\title{
Reflected shock bifurcation in a square channel
}

\author{
A. M. Khokhlov* \\ The University of Chicago, Chicago, IL \\ J. M. Austin ${ }^{\dagger}$ \\ University of Illinois, Urbana, IL \\ C. Bacon, S. Aithal, and K. Riley \\ Argonne National Laboratory, Chicago IL
}

\begin{abstract}
We examine the bifurcation of a reflected shock in a three-dimensional, square channel through numerical simulations using a distributed memory parallel adaptive mesh refinement, Navier-Stokes solver with multicomponent equation of state and microscopic transport. The three-dimensional, rather than axisymmetric, geometry introduces considerable complexity into the flow structures, particularly in the vicinity of the corners. Spanwise cross-sections show the boundary layer growth is significantly reduced in the corner regions, where the shape of the turbulent jet and recirculation regions are modified. This appears to be consistent with existing experimental studies which report the boundary layer in the corner region is deformed and the propagation velocity is reduced. A pair of triple points and a diagonally-oriented Mach reflection with shear layers directed towards the corner apex are also observed in the spanwise view.
\end{abstract}

\section{Introduction}

The interaction of a reflected shock wave with the boundary layer produces complex, unsteady, threedimensional effects including shock bifurcation, formation of recirculation bubbles and turbulent jets. The magnitude of the effects depends on the input physics, tube geometry, and wall boundary conditions including wall roughness and heat conduction through the walls. The details of shock reflection are also dependent on the development and growth of a boundary layer behind the incident shock. All of these processes may have a varying degree of influence on shock tube experiments measuring ignition delay, species concentration history, and kinetic rates. Shock tube data are typically compared with calculations that assume a zero-dimensional, constant volume adiabatic explosion. However, three-dimensional effects such as hot-spots generated in the recirculation zone associated with the bifurcated structure can lead to discrepancies between experiments and simulations, see Davidson and Hanson ${ }^{7}$ for example. Three-dimensional channel flows are challenging to probe with single point, wall-mounted transducers or line-of-sight optical diagnostics typically used in ignition delay experiments. Minimizing the impact of the shock bifurcation can reduce restrictions on large facility diameters, increase access to lower temperature conditions with longer test times, and reduce dilution with large amounts of gas with high specific heat.

The first documented experimental observation of the shock bifurcation phenomena was by Mark, ${ }^{15}$ who established the criterion that the bifurcation occurs when the stagnation pressure of the boundary layer is less than the normal reflected shock pressure; the boundary layer fluid is unable to pass through the normal reflected shock. Mark's model was restricted to perfect gas flows $(\gamma=1.4)$, for which a large increase in foot angle (angle between bifurcated foot and shock tube wall) is predicted for large Mach numbers. Davies and Wilson were the first to explore the validity of Mark's model at high Mach numbers. ${ }^{8}$ Their study included

\footnotetext{
*Professor, Department of Astronomy and Astrophysics

${ }^{\dagger}$ Assistant Professor, Department of Aerospace Engineering, Senior Member AIAA

${ }^{\ddagger}$ Argonne Leadership Computing Facility, Mathematics and Computer Science Division
} 
measured growth rates of the bifurcated foot and discussed the effects of transition. Bull and Edwards also experimentally investigated the reflected shock and boundary layer interaction region; their study was primarily concerned with the influence of driver gas contamination upon bifurcation velocities. ${ }^{3}$ Matsuo et al. measured the growth rate of the bifurcation region from schlieren images, for which they saw fair to good agreement with their numerical model. ${ }^{16}$ They also measured reflected shock velocities for comparison with their model. Strehlow and Cohen observed boundary layer interaction with the reflected shock for all gases tested except helium and argon. ${ }^{18}$ Their efforts concentrated upon measurement of reflected shock velocities, for which they saw the largest deviation away from one-dimensional theory with bifurcation. Strehlow and Cohen were the first to observe the loss of self-similarity in bifurcated foot growth rate and its growth towards an asymptotic height. Byron and Rott showed that if the ratio of specific heats behind the reflected shock was less than 1.54 then a minimum Mach number exists above which a strong laminar boundary layer interaction will always occur. ${ }^{5}$ Dyner found that relative bifurcated foot velocities increased with increasing reflected shock Mach numbers and decreasing specific heat ratios. ${ }^{9}$ Bifurcation heights at Mach 3 for air and $\mathrm{CO}_{2}$ were measured by Fokeev and Gvozdeva. ${ }^{10}$

More recently, Petersen and Hanson investigated the extent and magnitude of the bifurcation region for a variety of different conditions. ${ }^{17}$ Mixture specific heat ratios ranged from 1.29 to 1.51 , molecular weights from 14.7 to 44.0 and reflected shock pressures and temperatures from 11 to 265 atm and 780 to $1740 \mathrm{~K}$ respectively. Based upon their findings, they presented empirical relations for the size (step height) of the bifurcation region. These correlations indicated that the bifurcation characteristic dimensions were most dependent upon shock velocity and specific heat ratio, specifically that increasing the velocity and decreasing the specific heat ratio has the effect of increasing the bifurcation region. Bifurcation dimensions were measured via laser-schlieren visualization and shock velocities measured using fast-response pressure transducers.

The above experiments were all conducted in shock tubes with circular cross-section. There is experimental evidence that the flowfield is substantially more complex near the corners of a square tube. The experiments of Brossard et al. ${ }^{2}$ were carried out in a facility with a test section of $72 \times 72 \mathrm{~mm}$ initial cross-section. A sharp plate was used to reduce the width of the test section to 56, 22, and $8 \mathrm{~mm}$. Test gases were nitrogen, air, and carbon dioxide with initial pressures from 0.0065 to 0.046 atm and incident shock Mach numbers from 2.5 to 4. Static wall pressure measurements were made. Schlieren images show a complex three-dimensional structure during shock reflection. A typical bifurcated reflection occurs, qualitatively the same as in axisymmetric geometries. In addition, deformation and retardation of the boundary layer separation line is observed near the channel corner. The curvature of the separation line along the wall observed in the schlieren images is consistent with observations from our present simulations. In the experiments, the length of the deformed region was reported to be on the order of the bifurcation foot height. The experimentally-measured pressure at the separation point did not agree with calculated values using Mark's model.

Numerical simulations of shock bifurcation in a rectangular tube were carried out by Gamezo et al. ${ }^{11}$ The focus of this study was reactive mixtures and shock-flame interactions, with the initial condition consisting of an initially ellipsoid flame. A complex and unsteady reactive shock bifurcation structure was observed, with the recirculation zone acting as a flame-holding structure. The main three-dimensional effect due to the rectangular channel was the interaction of two reactive shock bifurcations from adjacent walls to produce an oblique Mach stem. The oblique Mach stem was then observed to interact with the central Mach stem to create a hot-spot which led to detonation of the front.

In the present study, we build on these previous works and examine in detail three-dimensional shock bifurcation in a square channel. The shock bifurcation structure is known to be sensitive to the tube geometry, as discussed above, and the outstanding issues that motivate this study include: What are the mechanisms and effects of accelerated boundary layer separation in corners, as observed in the experiments of Brossard et al.? What is the impact of channel geometry on turbulent statistics of the flow and subsequently the bifurcation, jet, and recirculation structure? Key features of the present simulations are very high resolution (on the order of 100 times previous studies) inside the boundary layer, and the inclusion of temperaturedependent transport properties. The large range of physical scales which are relevant to shock-boundary layer interactions present a considerable numerical challenge. Temperature-dependent transport increases the difficulty of numerical convergence. The improved resolution and more advanced physical models are made possible through the use of a distinctive computational algorithm, as described in detail in Section II.A. 


\section{HSCD code}

Numerical simulations are carried out using a distributed memory parallel adaptive mesh refinement (AMR) reactive flow Navier-Stokes solver ${ }^{13,14}$ which we augmented with the multicomponent equation of state (EOS), and microscopic transport. Euler fluxes are calculated using a second-order accurate, Godunovtype, conservative scheme with a Riemann solver ${ }^{6}$ and a monotone VanLeer reconstruction. ${ }^{19}$ Viscous, mass diffusion, and heat fluxes are calculated using second-order central differencing. Equation of state implementation is based on the NASA 7 terms polynomial fits. ${ }^{4}$ Transport coefficients for mixture viscosity are calculated according to Wilke ${ }^{20}$ modified by Bird. ${ }^{1}$ Formulae for heat and mass diffusion coefficients were adopted from Hirschfelder et al. ${ }^{12}$ The Lennard-Jones 6-12 collisional integrals required for calculations of transport coefficients were fit with 7-th order polynomials over reduced temperatures from 0.7 to 100 .

A distinct feature of the code is a highly dynamic cell-by-cell AMR based on a parallel fully threaded tree (FTT) structure. In ordinary trees pointers are directed from parents to children. In FTT, the pointers are inverted and directed from groups of children to parents and parent's siblings. This arrangement eliminates expensive tree searches which are notoriously difficult to parallelize and it allows all operations, including mesh refinement and de-refinement, to be performed in parallel. The mesh is refined around shocks, discontinuities and in regions containing large gradients of physical variables such as chemical variables, temperature, vorticity, and so on. AMR is performed every fourth time step after which the cells are rebalanced across the processors using a heuristic to estimate the amount of work required by the cells, maintain data locality, and minimize communications across MPI ranks. The FTT library automatically synchronizes the data in ghost cells after global operations. The code can run in the uniform grid and static mesh refinement modes as well. The code was run in a hybrid OpenMP/MPI mode and showed good scaling performance on more than $100 \mathrm{~K}$ computer cores on the BG/P.

\section{II.A. Computational Algorithm}

Architecturally, the code consists of three separate layers: (1) the FTT library which provides general services related to all parallel aspects of the code's execution; (2) the reactive Navier-Stokes AMR code, ALLA, which rides on top of FTT and is responsible for numerical integration and AMR; (3) problemspecific algorithms such as material properties routines (equation of state, kinetics, microscopic transport) and problem initialization routines called from ALLA.

The FTT library contains a parallel implementation of a multi-level adaptive computational mesh. All parallel aspects of computations are hidden inside the library. To a user, FTT provides a shared view of the mesh with added global services such as I/O, automatic and on-demand load balance, data synchronization across MPI processors, functions for mesh refinement, and iterators for global parallel operations of the mesh. The memory overhead of parallel FTT in 3D is $\simeq 2.6$ integers per cell, significantly less than 8 integers per cell for an oct-tree, or the 14 integers per cell for a threaded oct-tree. A space-filling curve approach is used in FTT for domain decomposition. A detailed description of parallel aspects of FTT is given in reference ${ }^{14}$

Computations in ALLA are organized as a set of global computational steps, with each step followed by communication work which synchronizes data across the domain decomposition boundaries. Application algorithms are programmed in terms of work-functions which are passed to and executed by the global parallel iterator. On each MPI rank the iterator parses the mesh and passes small chunks of cells to a work-function until the entire mesh is processed. The loop over the cells in a chunk is performed inside the work-functions themselves. Physical quantities in fluid dynamic part of the code are updated on an interfaceby-interface basis. First total fluxes of physical quantities across an interface are computed, then physical quantities in adjacent cells updated, and the fluxes are immediately discarded. This guarantees conservation of all extensive physical quantities while avoiding unnecessary inter-grid synchronization stages and their associated memory and communication overheads. The mesh can be refined around shocks, discontinuities and in regions containing large gradients of physical variables such as chemical variables, temperature, vorticity, and so on. AMR is performed every fourth timestep after which the cells are rebalanced across the processors using a heuristic to estimate the amount of work required by the cells. This typically results in small incremental movements of data across domain decomposition boundaries. A schematic of the simulation domain is shown in Figure 1. 


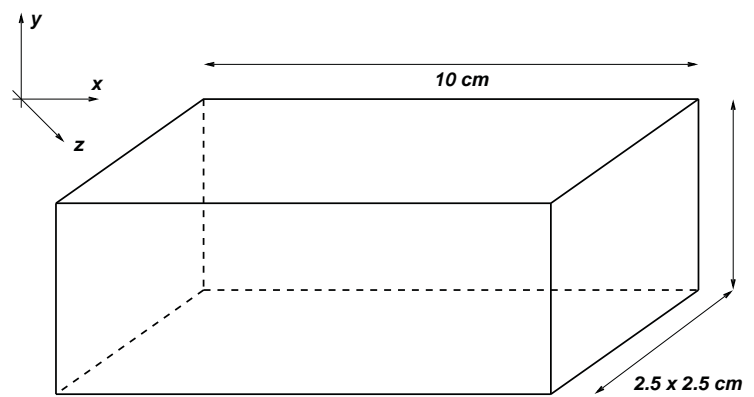

Figure 1: Schematic of the simulation domain and axes orientation. The incident shock propagates left to right.

\section{Results}

The HSCD code described in previous sections has been used to model a process of shock reflection in a square tube section $10 \times 2.5 \times 2.5 \mathrm{~cm}^{3}$ with a closed end wall. The computational setup is shown in Figure 1. Non-slip adiabatic wall boundary conditions were imposed on the side walls and the end wall of the tube. Zero-gradient inflow boundary conditions were imposed on the left boundary. Navier-Stokes calculations were performed for a $2 \mathrm{H}_{2}+\mathrm{O}_{2}$ gaseous mixture initially at 0.1 atm pressure. The incident shock wave had a Mach number $\mathrm{M}=3$. We used an AMR mesh with maximum resolution of greater than 10 microns. The uniform equivalent resolution would have required about $N_{\text {cell }} \geq 6.25 \times 10^{10}$ computational cells. Computations were performed for $1 / 4$ of the tube cross-section assuming symmetry with respect to the centerline. The AMR calculation used about $N_{\text {cell }} \geq 5 \times 10^{8}$ computational cells on an average. The mesh was refined around shocks, contact discontinuities, pressure and density gradients, and in regions of high vorticity. The latter allowed the scheme to detect and keep at the maximum resolution the turbulent recirculation region which forms near the walls behind the reflected bifurcated shock structure.

Figures 2 - 6 show the results of the simulation of a reflected shock bifurcation, at 0.1 atm initial pressure. The incident shock with Mach number $M=3$ initially propagated inside a square shock tube from left to right towards the solid end wall. The flow behind the shock generated a thin boundary layer. Eventually the incident shock reflected from the wall and started to propagate backwards. Interaction of the reflected shock with the boundary layer leads to shock bifurcation: An oblique shock developed near the side walls, the boundary layer separated from the wall, and a growing low pressure recirculation bubble formed, with a turbulent jet moving along the side walls towards the oblique shock. The turbulence in the recirculation jet was of very high intensity, with fluctuation velocities approaching the sound speed. Acoustic waves were generated and observed to emanate from this region.

Figure 3 shows three consecutive time frames of a reflected shock bifurcated structure after the reflection occurs. The reflected shock moves to the left. The height of the bifurcated shock foot was observed to grow with time. Figure 4 shows a typical cross-section of the dynamic computational mesh during an instant of the simulation.

The simulation reveals intriguing features near the corners of a square tube. Spanwise cross-sections of the developing flow are shown in Figure 5. A three-dimensional Mach stem structure due to interaction of the two systems of bifurcated shocks, one for each wall, is evident. The oblique shocks interact with the boundary layers in the vicinity in the corner. There is a region of relatively low-speed fluid behind the Mach wave. A considerable reduction in boundary layer thickness is observed in the corner region in this cross-sectional view. Figure 6 shows the plan-view cross-section of the temperature field at a 20 micron distance from the corner. Figure 6 illustrates a significant inhomogeneity and a strong influence of the presence of a corner on the structure of the recirculation bubble. As evidenced by the figure, the presence of the corner delays the formation of turbulence and changes the shape of the turbulent jet and the recirculation region. This behavior appears to be consistent with the experiments of Brossard et al. ${ }^{2}$ who found that near the corner the separation region was deformed and propagated with lower velocity, with the length of the deformed region being of the order of the bifurcation foot height. 


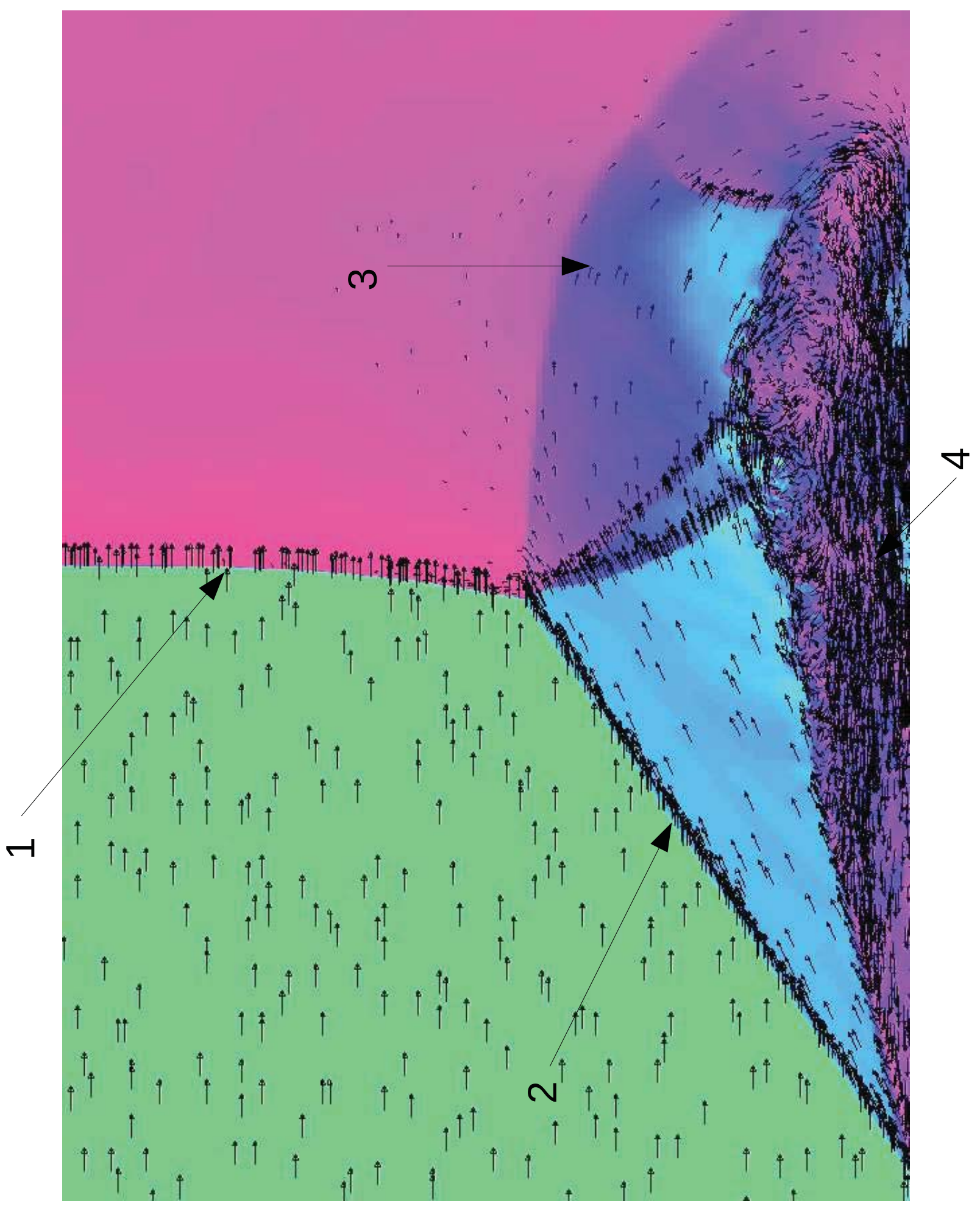

Figure 2: Bifurcation of a reflection of a Mach 3 shock. Temperature with superimposed velocity vectors in a two-dimensional XY-plane located $2.5 \mathrm{~mm}$ from the side wall of the tube at $3.55 \mu \mathrm{sec}$ after reflection. The field of view is approximately $3 \times 2.5 \mathrm{~mm}^{2}$. 1 - primary reflected shock; 2 - oblique reflected shock; 3 recirculation region; 4 - turbulent jet. Colors correspond to $\sim 700 \mathrm{~K}$ - green, $1100 \mathrm{~K}$ - blue, and $1500 \mathrm{~K}$ - red. 

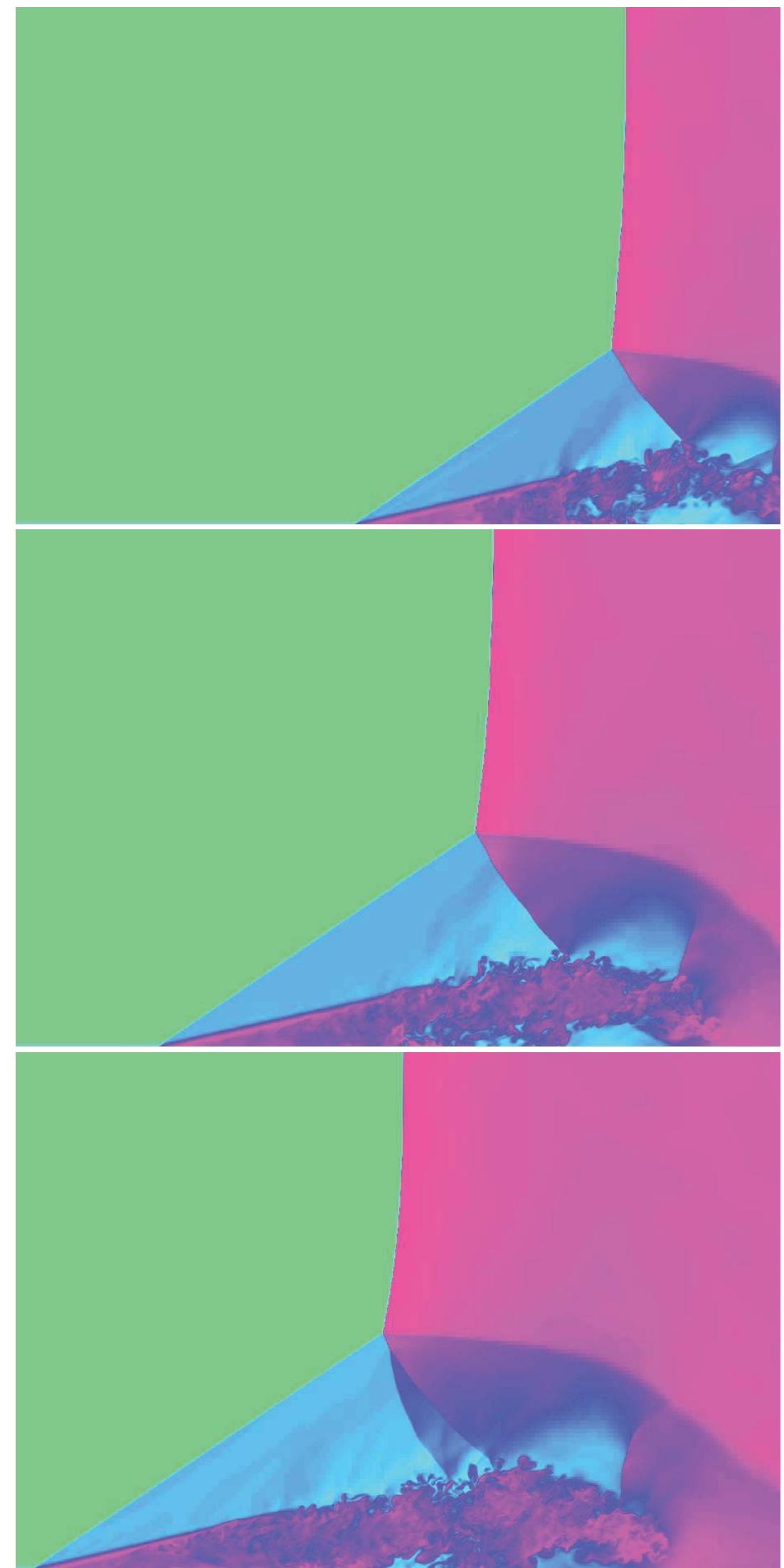

Figure 3: Reflection of a Mach 3 shock, similar to Fig. 2. Frames correspond to 2.04, 2.96, $3.55 \mu$ sec after reflection. 


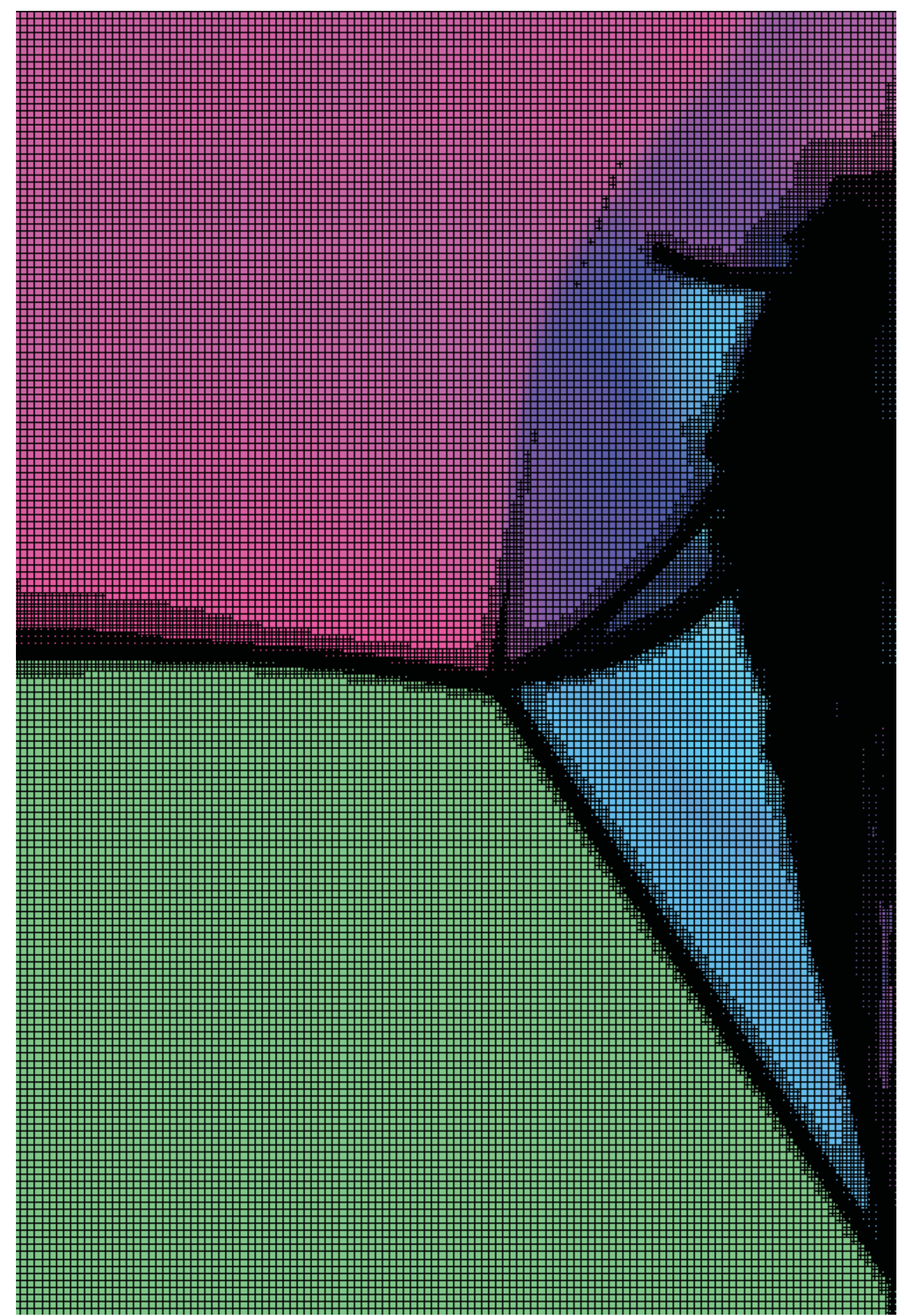

Figure 4: Cross-Section of an AMR computational mesh superimposed on the temperature field shown in Fig. 3. The minimum $d x$ of the simulation is 2.44 microns.

\section{Acknowledgments}

This work was supported through the Basic Energy Sciences and Advanced Scientific Computing Research programs of the Department of Energy Office of Sciences under contract DE-SC0002954. This research 


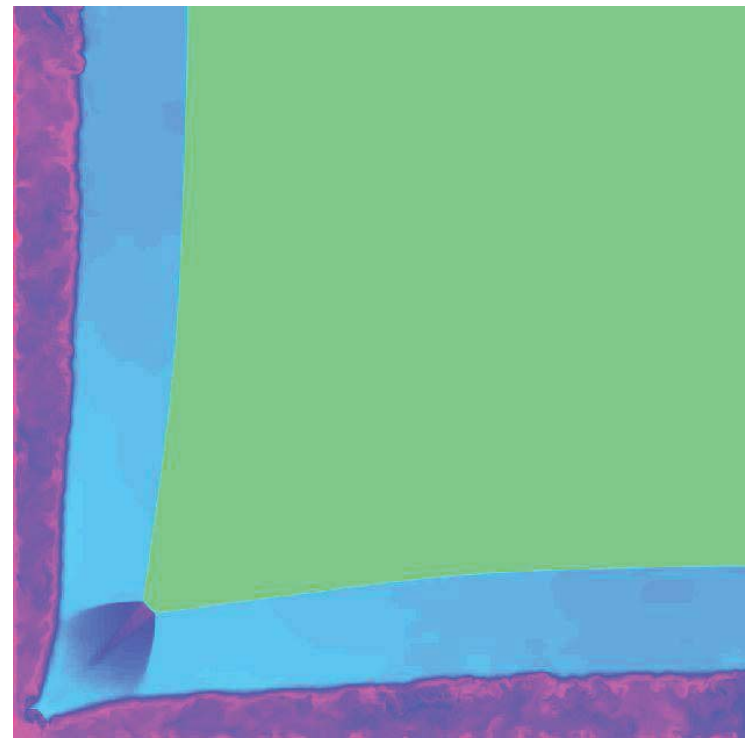

a)

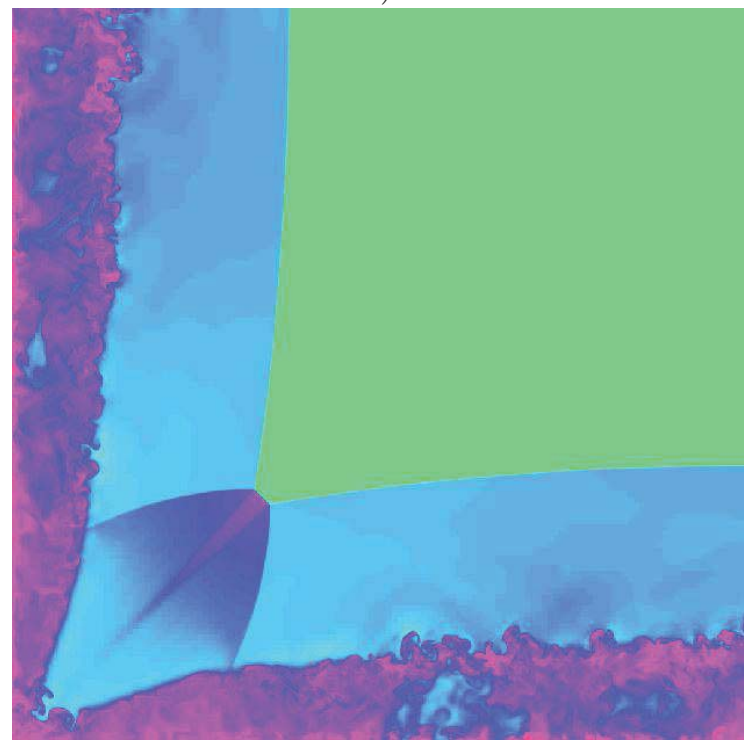

c)

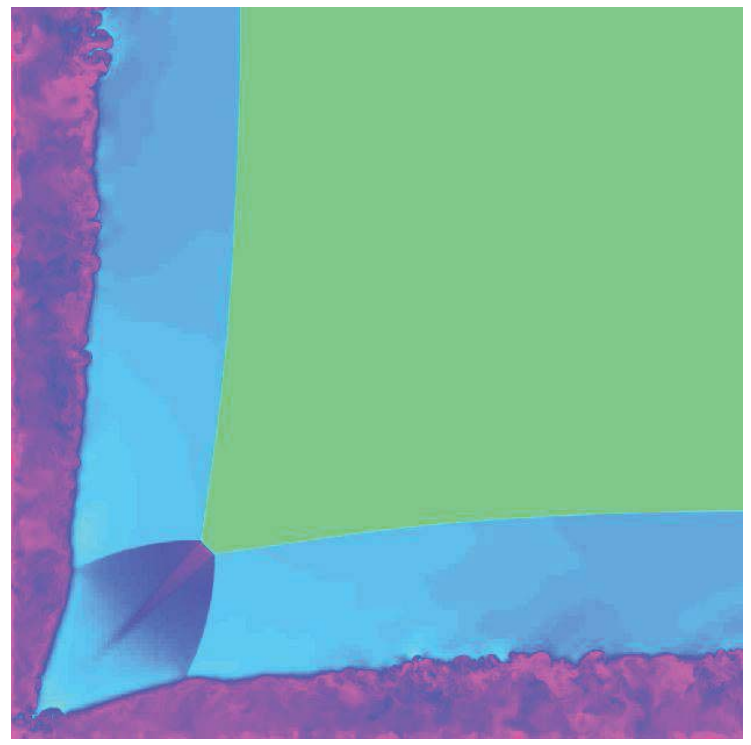

b)

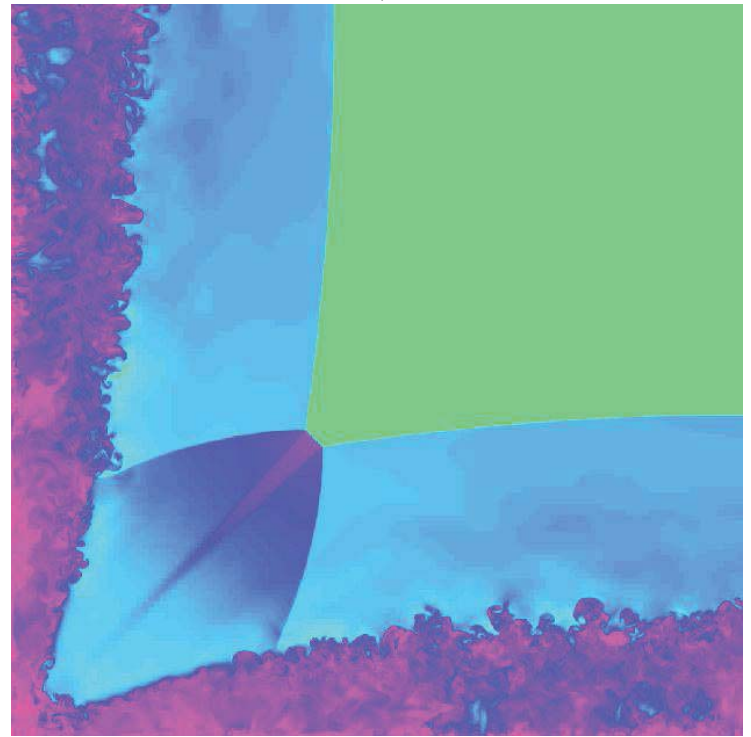

d)

Figure 5: YZ crossections of the developing flow showing the three-dimensional structure of the bifurcated shock. 


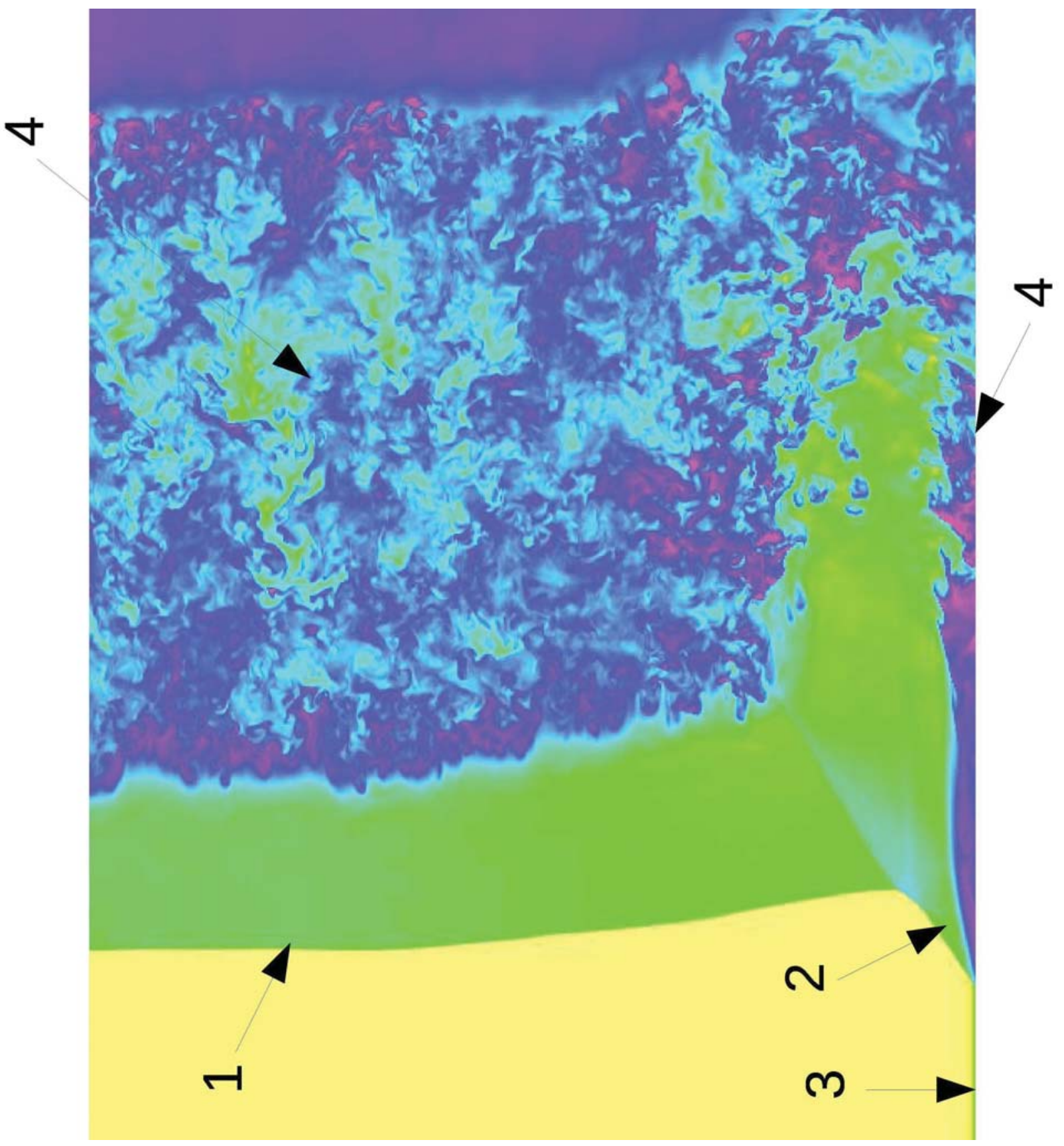

Figure 6: Reflection of a Mach 3 shock. Cross-Section of the temperature field at a 20 microns distance from the corner. 1 - initial boundary layer; 2 - primary reflected shock; 3 - oblique reflected shock; 4 - turbulent recirculation flow.

used resources of the Argonne Leadership Computing Facility at Argonne National Laboratory, which is supported by the Office of Science of the U.S. Department of Energy under contract DE-AC02-06CH11357. AK acknowledges partial NSF support through AST-0709181 and TG-AST090074 grants. The authors thank Dr. Barry Smith (MCS Division of the Argonne National Laboratory)) for helpful advice, and thank Manu Sharma at the University of Illinois for his contributions to this study. 


\section{References}

${ }^{1}$ R. B. Bird, Stewart W. E., and Lightfoot E. N. Transport Phenomena. John Wiley and Sons, 1960.

${ }^{2}$ J. Brossard, N. Charpentier, T. V. Bazhenova, V.P. Fokeev, A. A. Kalachev, and A.I. Kharitonov. Experimental study of shock wave reflection in a narrow channel. In Proceedings of the 15th International Symposium on Shock waves and Shock Tubes, pages 163-169, 1984.

${ }^{3}$ D.C. Bull and D.H. Edwards. An investigation of reflected shock interaction process in a shock tube. AIAA J., 6(8):1549, 1968.

${ }^{4}$ A. Burcat and B. Rucic. In Third Millenium Ideal Gas and Condensed Phase Thermochemical Database for Combustion with Updates from Active Thermochemical Tables, 2005.

${ }^{5}$ S. R. Byron and N. Rott. In Proceedings of the 1961 Heat transfer and Fluid Mechanics Institute, page 38, 1961.

${ }^{6}$ P. Colella and H.M. Glaz. Efficient solution algorithms for the riemann problem in real gases. J. Comput. Phys., 59:264, 1985.

${ }^{7}$ D. F. Davidson and R.K. Hanson. Recent advances in shock tube/laser diagnostic methods for improved chemical kinetics measurements. Shock Waves, 19(4):271-283, 2009.

${ }^{8} \mathrm{~L}$. Davies and J. Wilson. Influence of reflected shock and boundary-layer interaction on shock-tube flows. Physics of Fluids Supplement, 1969.

${ }^{9}$ H. Dyner. Density variation due to reflected shock-boundary-layer interaction. Physics of Fluids, 9(5):879-892, 1966.

${ }^{10} \mathrm{~V}$. Fokeev and L. Gvozdeva. Study of bifurcation of reflected shock waves in channels of various cross-sections. 17th International Symposium on Shock Waves and Shock Tubes, 1990.

${ }^{11}$ V. N. Gamezo, E. S. Oran, and A.M. Khokhlov. Three-dimensional reactive shock bifurcations. In Proceedings of Combustion Institute, pages 1841-1847, 2005.

${ }^{12}$ J.O. Hirschfelder, C.F. Curtiss, and R.B. Bird. Molecular theory of gases and liquids. Elsevier, 1954.

${ }^{13}$ A.M. Khokhlov. Fully threaded tree algorithms for adaptive refinement fluid dynamics simulations. J. Comp. Phys., 143:519, 1998

${ }^{14}$ A.M. Khokhlov and A.Yu. Chtchelkanova. Fully threaded tree algorithms for massively parallel computations. In Proceedings of the Ninth SIAM Conference on Parallel Processing for Scientific Computing, San Antonio, Texas, USA, March 22-24, 1999.

${ }^{15} \mathrm{H}$. Mark. The interaction of a reflected shock wave with the boundary layer in a shock tube. NACA Technical Memo, 15:333-340, 2006.

${ }^{16} \mathrm{~K}$. Matsuo, K. Shigetoshi, and K. Kazuyuki. Interaction of a reflected shock wave with the boundary layer in a shock tube. Bulletin of the JSME, 17(110):1039-1046, 1974

${ }^{17}$ E L. Petersen and R K. Hanson. Measurement of a reflected-shock bifurcation over a wide range of gas composition and pressure. Shock Waves, 15:333-340, 2006

${ }^{18} \mathrm{R}$. Strehlow and A. Cohen. Limitations of the reflected shock technique for studying fast chemical reactions and its application to the observation of relaxation in nitrogen and oxygen. Journal of Chemical Physics, 30:257-265, 1959.

${ }^{19} \mathrm{~B}$. van Leer. Towards the ultimate conservative scheme. V. A second-order sequel to Godunov's method. J. Comput. Phys, 54:101, 1979.

${ }^{20}$ Wilke. A viscosity equation for gas mixtures. J. Chem. Phys., 18:517-519, 1950. 